NASA Technical Memorandum 105274

$1 N-32$

AIAA-91-3423

\title{
Evaluation of Components, Subsystems, and Networks for High Rate, High Frequency Space Communications
}

Robert J. Kerczewski, William D. Ivancic, and John E. Zuzek

Lewis Research Center

Cleveland, Ohio

. Prepared for the

Conference on Advanced Space Exploration Initiative Technologies

cosponsored by AIAA, NASA, OAI

Cleveland, Ohio, September 4-6, 1991 


(1)
0
$=$

$\sim-$ 


\title{
EVALUATION OF COMPONENTS, SUBSYSTEMS, AND NETWORKS FOR HIGH RATE, HIGH FREQUENCY SPACE COMMUNICATIONS
}

\author{
Robert J. Kerczewski", William D. Ivancic, and John E. Zuzek* \\ NASA Lewis Research Center \\ Cleveland, Ohio
}

\begin{abstract}
The development of new space communications technologies by NASA has included both commercial applications and space science requirements. At NASA's Lewis Research Center, methods and facilities have been developed for evaluating these new technologies in the laboratory. NASA's Systems Integration, Test and Evaluation (SITE) Space Communication System Simulator is a hardware-based laboratory simulator for evaluating space communications technologies at the component, subsystem, system, and network level, geared toward high frequency, high data rate systems. The SITE facility is well-suited for evaluation of the new technologies required for the Space Exploration Initiative (SEI) and advanced commercial systems. This paper describes the technology developments and evaluation requirements for current and planned commercial and space science programs. Also examined are the capabilities of SITE, the past, present, and planned future configurations of the SITE facility, and applications of SITE to evaluation of SEI technology.
\end{abstract}

\section{Introduction}

To enable various potential future commercial services, and to meet NASA's space science communication needs such as future TDRSS-type data relay and SEI communication systems, numerous technology development programs exist or are planned. These technologies include $\mathrm{Ka}-\mathrm{B}$ and and millimeter-wave frequency transmit and receive hardware, multi-beam antenna technology, on-board switching and processing, high rate bandwidth-efficient modulation and coding, microwave, millimeter-wave, and optical intersatellite links (ISL), cost-efficient ground terminals, and network implementation and control.

At NASA's Lewis Research Center, we are concerned with methods for evaluating these new technologies in the laboratory as they are developed, and performing simulation in hardware at the network and system level in order to both evaluate and extend the development of systems and subsystems.

In the following sections, some of NASA's current and planned future commercial and space science technology evaluation requirements will be described. The development of the SITE Space Communication System Simulator will be reviewed, including current projects and future plans which include MMIC evaluation, intersatellite link networking, and on-board switching and processing. Application of the SITE facilities and evaluation techniques to advanced SEI communication system technologies will be discussed.

\section{Space Communication Technology Trends}

In commercial communications systems, Lewis Research Center has been involved in the development of the Advanced Communications Technology Satellite (ACTS), a Ka-Band multi-beam satellite system which includes on-board IF and baseband switching and processing as the heart of a time division multiple access (TDMA) network. This work is now being followed

* Member, AIAA

Copyright 1991 by the American Institute of Aeronautics and Astronautics, Inc. No copyright is asserted in the United States under Title 17, U.S. Code. The U. S. Government has a royalty-free license to exercise all rights under the copyright claimed herein for Government purposes. All other rights are reserved by the copyright owner. 
other by advanced commercial concepts, such as FDMA-TDMA systems including advanced on-board switching and processing, MMIC-based satellite hardware, low-cost ground terminal development, and improved modulation and coding.

These systems require the development of advanced components and subsystems. Medium and high power amplifiers at frequencies up to $30 \mathrm{GHz}$ are needed for both space and ground applications, using electron tube (TWT) and solid state technology. Higher efficiency and reliability is the goal for the space-based segment, while low cost is the primary driver in ground terminal hardware. Low noise receivers also fit these general requirements.

Components for IF switching and subsystems for baseband switching and processing are also currently in development. For IF switching, as well as receiver and other analog processing functions, monolithic microwave integrated circuit (MMIC) technology is being applied. Digital signal processing techniques, high speed electronic circuits, and optical processing are being developed for digital beamforming and on-board demodulation/remodulation functions. The development of advanced modems and codecs, especially at higher data rates ( $>200 \mathrm{Mbps}$ ) is being driven by the need to transfer large volumes of data in short periods of time over limited access systems such as medical, scientific, and supercomputer networks.

Other subsystems which must be developed and verified include the ground terminal, which can be subdivided into transmit and receive sides as well as RF and digital portions. Evaluation of transmit, receive, and IF/baseband processing portions of the satellite transponders is also necessary.

As satellite systems are required to perform more and more switching and processing tasks, the role of network control increases in importance. It is necessary to test the network's response in initial acquisition and tracking, controlling entrance and exit of users to the system, controlling and monitoring the satellite processing hardware, and responding to system perturbations such as interference, rain fade, and satellite range variations.

The complexity and evolutionary aspects of the potential Space Exploration Initiative program offer a tremendous number of technological challenges in the areas of
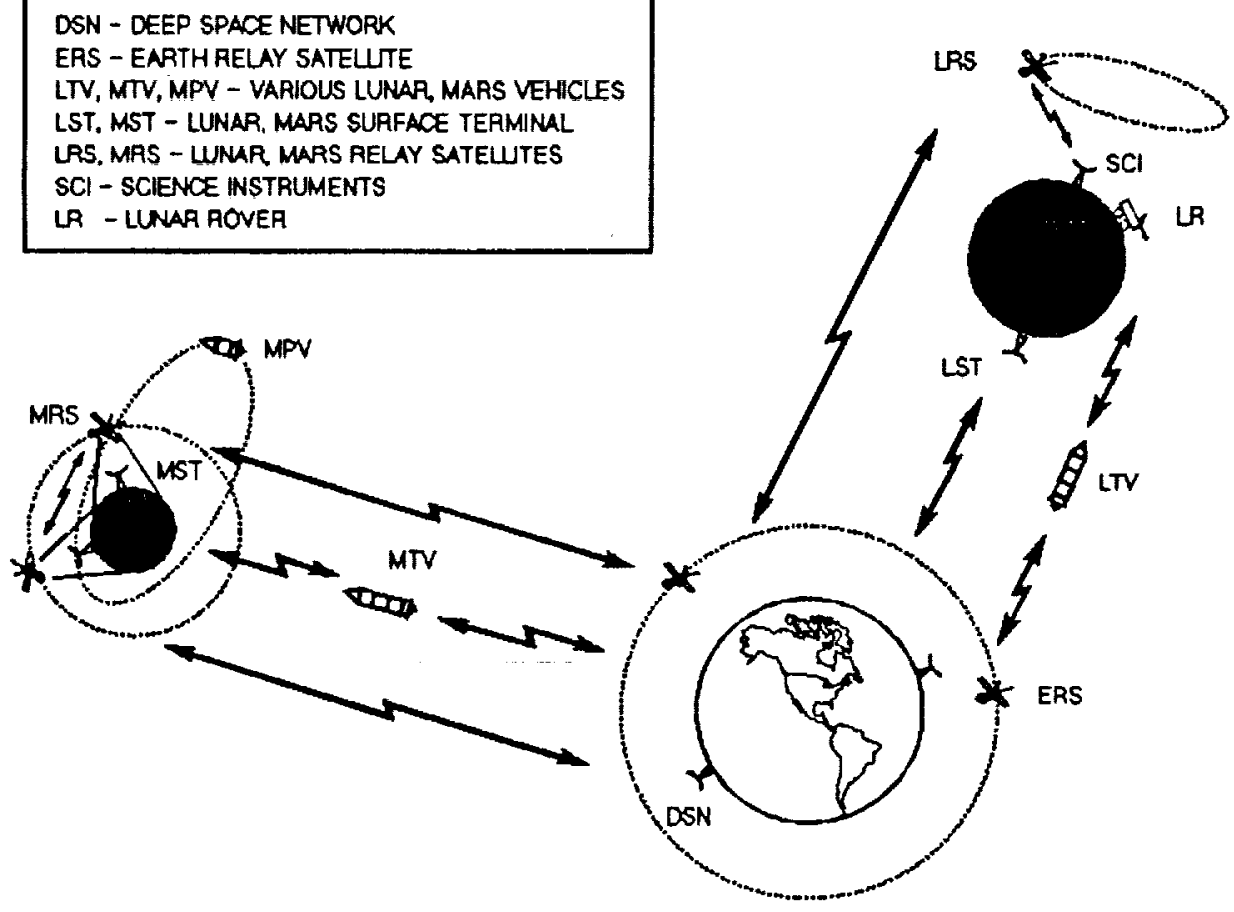

Figure 1 - Space Exploration Initiative Communication System Concept 
telecommunications and information management. The SEI system may eventually include lunar and Mars surface terminals, a variety of science instruments, astronauts, rovers, robotic elements, and in-orbit facilities such as piloted vehicles and lunar and Mars relay satellites ${ }^{1}$ as exemplified by Figure 1. Many of these nodes will be linked with high data rate long range links which will require an evolution from Ka-band frequencies to millimeter-wave frequencies and possibly to optical transmissions for certain links. Relay satellites will need to squeeze every conceivable $d B$ out of the links requiring advances in efficiencies and designs for many technologies. As the network evolves, its complexity, connectivities, and technologies must also evolve.

One key technology will be very low noise $(1.0-1.5 \mathrm{~dB}$ or less) space-based receivers. These demands may suggest constraints on various receiver subsystem components as well. Another key subsystem will be the space-based antennas. It is likely that phased array antennas probably based on MMIC technologies will be required for some communications nodes. In addition, frequencies of $\mathrm{Ka}$-band and higher will place new demands on the antenna subsystems and feed network components. Additional efficiencies might be found in the integration of optical technologies such as optical interconnects or beam forming networks into the antenna system. Very low loss feed systems will be required in many cases.

Beam switching will also be required with technologies similar to or possibly beyond ACTS. Another implication of this switching would be a baseband processor and/or microwave switch matrix for routing signals. Other components such as amplifiers, both electron beam (TWTA) and solid state (SSPA), would need to be tested and evaluated, especially those with advances in efficiency or power and those designed to operate at millimeter-wave frequencies. Another possibility for development and testing would be modems and codecs. Modems may have special requirements in that different SEI links may require different modulation schemes since some links will be bandwidth-limited while others, such as the Mars return links, will be severely power-limited. This translates to the use of QPSK or some other M-ary PSK scheme for most links and the use of some high order FSK modulation scheme or advanced trellis-coded modulation for the Mars return links. Cross-strapping between these two networks may cause additional difficulties. Also, the SEI communications links would likely place tremendous demands on the error detecting/correcting capabilities of the subsystems compared to conventional Earth orbiting systems. A wide range of requirements are likely with bit error rates from $10^{-3}$ for voice to as low as $10^{-12}$ for essential telemetry or science data. The latter requirement may be necessitated by any sort of data compression scheme which require more stringent error control. Finally, various data compression algorithms will be utilized and the hardware for these will need to be integrated and tested in the subsystems and systems in which they will operate.

The demands the SEI missions will place on the telecommunications and information management networks will be similar to those imposed on an Earthbased communications network except that the resources must be managed in space. It is likely that the system will evolve into a complex network which must be able to allocate channels and communications resources on demand or even autonomously. Thus, some sort of minimally attended network management system including an artificial intelligence based network planner/scheduler may be needed to deal with these resources. Additionally, network and component fault tolerance will be of paramount importance in these missions. The networks themselves will have to be able to detect and isolate faults and repair them or work around them as is necessary.

Link impairments and the systems responses to them will have to be modelled and evaluated. Such impairments will include atmospheric attenuation, pointing errors due to strict antenna pointing accuracies, network and component outages, and occultations or other unforeseen anomalies. It will also be necessary to test various communications protocols for the data systems and potentially integrate space-based processors and data storage devices into the total system.

\section{Technology Evaluation Approach}

The communications technologies must be evaluated at four levels: component, subsystem, system, and the network level.

The dividing line between components and subsystems is somewhat arbitrary. Thus, they have similar test requirements. Important information regarding the electrical performance can be gained through bench-top 
testing of stand-alone hardware. However, placing the component in a system environment and using both unmodulated and modulated test signals gives considerable information on the component's or subsystem's performance in transmitting real signals, as well as its effect on the overall system. The effect of a component or subsystem on the bit-error rate (BER) of the system can be measured and compared with other components, different designs, varying operating conditions, or with the component or subsystem bypassed. The effect of a component or subsystem on the operation of the network can only be measured by placing it in a portion of a network containing at least the minimum number of nodes required for an accurate network model.

Some components or subsystems require other portions of the system for proper evaluation. Components such as codecs require modems for performance measurement, and the modems require noise and signal variation and calibration as well as BER measurement systems, all of which can be made part of the overall system test environment. The switching subsystem for an on-board processing satellite requires a number of test signals which must resemble the real signals in the system. In addition, interfaces between these major subsystems and between the system and users require evaluation and verification. Such evaluation can only be performed in an end-to-end system environment.

Evaluation of systems must include the end-to-end performance of the system under all possible operating conditions. However, the testing must also take into account perturbations and distortions occurring over the entire communications link. Amplitude and phase distortions, whether induced by hardware or atmospheric propagation effects, noise, interference, and satellite range variation can affect system and network performance. The investigation and quantification of these effects and the methods developed to counteract them are of great interest. The test system can be designed to introduce these distortions and measure their effects.

Network control software and interfaces, switching, processing, and traffic handling algorithms, and overall system control methods can be developed and verified by simulating a minimum portion of a network in hardware. For a multi-beam satellite-switched TDMA (SS-TDMA) type system, for example, a minimum of three ground terminals located in three separate antenna spot beams is required. The development of such a simulator also allows a complete evaluation of the ground terminals to take place, since ground terminal acquisition, synchronization, tracking, and data transfer functions are heavily dependent on the network.

All of these requirements point to the need for a system and network oriented approach to space communications technology evaluation. Although the need for initial functional testing of components via standard bench top testing remains, nearly all of the other electrical performance testing requirements at each of the four technology levels can be met with an integrated test facility. In the next section, such a facility currently in use at NASA Lewis is described.

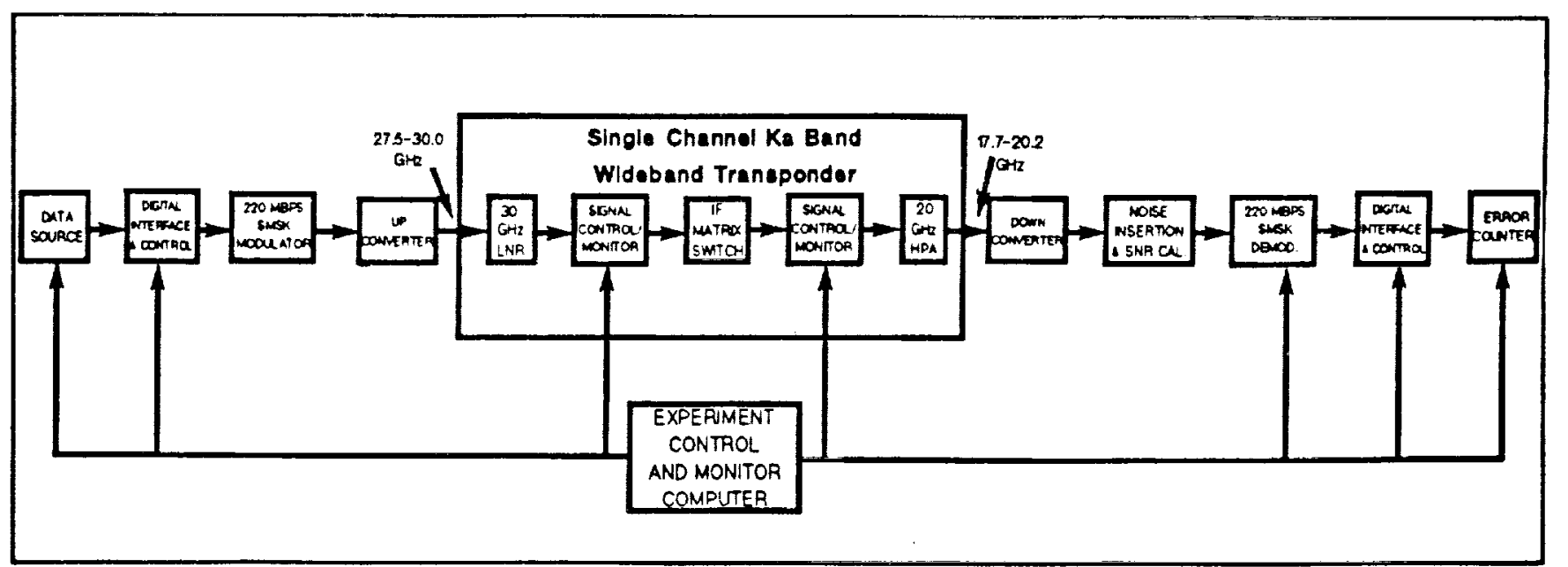

Figure 2 - SITE Space Communication System Simulator - Phase I 


\section{The SITE Space Communication System Simulator}

As mentioned above, the four technology levels can be evaluated in a single laboratory facility which includes the minimum amount of hardware required for assessing network operations combined with built-in monitoring. measurement, system control and experiment stimulation capabilities. The SITE evaluation facility includes all of these functions, under computer control, and has been used for evaluation of component, subsystem, system, and network technologies. In the initial configuration of the SITE facility, a single bent-pipe $30 / 20 \mathrm{GHz}$ high data rate satellite link was created. Further expansion and development in the past several years have lead to a three-terminal network simulation, with complete hardware links and multi-rate digital ground terminals. Plans are now being developed for the next phase of expansion, which will allow the evaluation of on-board switching and processing technologies currently under development, intersatellite link hardware and networks, and other satellite and ground terminal hardware and subsystems.

\section{Initial Configuration of SITE}

The SITE Space Communication System Simulator was originally conceived in $\mathbf{1 9 8 2}$ as a means of evaluating proof-of-concept components being developed for NASA's Advanced Communications Technology Satellite (ACTS) project ${ }^{2}$. The initial Phase I system consisted of a single $220 \mathrm{Mbps}$ satellite channel, operating at 30 $\mathrm{GHz}$ uplink and $20 \mathrm{GHz}$ downlink frequencies, shown in Figure 2. The system was able to measure bit-error rate (BER) as a function of $E_{b} / N_{o}$ using a continuous 220 Mbps MSK-modulated data stream.

The SITE Phase I system was used to evaluate Ka-Band components including solid state and TWT power amplifiers, low noise receivers, and matrix switches ${ }^{3-6}$. The performance of the satellite $30 / 20 \mathrm{GHz}$ transponder was evaluated ${ }^{7}$, as well as the effects of variable transponder output power for rain attenuation compensation $^{8}$, amplitude variations and equalization ${ }^{9}$, and group delay distortion ${ }^{10}$.

Although the Phase I system contained power, frequency, spectrum, and configuration monitoring, the most useful feature was the ability to automatically measure complete BER curves as a function of $E_{b} / N_{o}$. This was accomplished through the use of computer software and RF and digital hardware which allowed a pseudorandom data sequence to be generated, modulated, transmitted through the system under test, demodulated, and compared to a replica of the original data for error detection. Controlled amounts of noise are added and the $E_{b} / N_{o}$ is measured and incremented in $1 \mathrm{~dB}$ steps ${ }^{11-13}$.

\section{SITE Multi-Terminal Network Simulation}

Without eliminating the capabilities of the Phase I system, the SITE facility has been expanded in Phase II of the project to a three-terminal TDMA network, shown in Figure 3. This network includes three ground terminals, a three channel satellite transponder, seven simulated users, audio and video transmission, a radiative link to a remote terminal, and full network control capabilities.

The ground terminals currently allow continuous transmission at $220 \mathrm{Mbps}$ or bursted transmission at rates of 1 to $200 \mathrm{Mbps}$. Each ground terminal contains its own BER measurement and $E_{b} / N_{o}$ calibration, and thus can measure BER performance through any transponder path. As Figure 3 shows, the satellite matrix switch allows interconnectivity of any two ground terminals. Two ground terminals are equipped with three simulated users ${ }^{14}$, one of which interfaces to audio and video data. The third ground terminal, which can be located either in the third beam (third channel) or at the remote terminal, has a flexible user interface allowing numerous users at various rates.

Satellite range variation simulation is provided to allow a realistic timing environment for a TDMA system ${ }^{15}$. Satellite range and doppler shift affect the ability of ground terminals to synchronize with the network, and thus any system or network with dynamic switching must have this simulation capability.

For Ka-band systems, rain attenuation on both uplink and downlink portions is a primary concern. To allow evaluation of the effects on system performance, and on compensation techniques, methods for simulating realistic rain events have been included in the SITE facility ${ }^{16}$.

A remotely located ground terminal accessed through a radiative $30 / 20 \mathrm{GHz}$ link is also a part of the current SITE facility 17 . This terminal will demonstrate network control of a remote terminal, and will eventually be used to demonstrate a remote control and observation of 


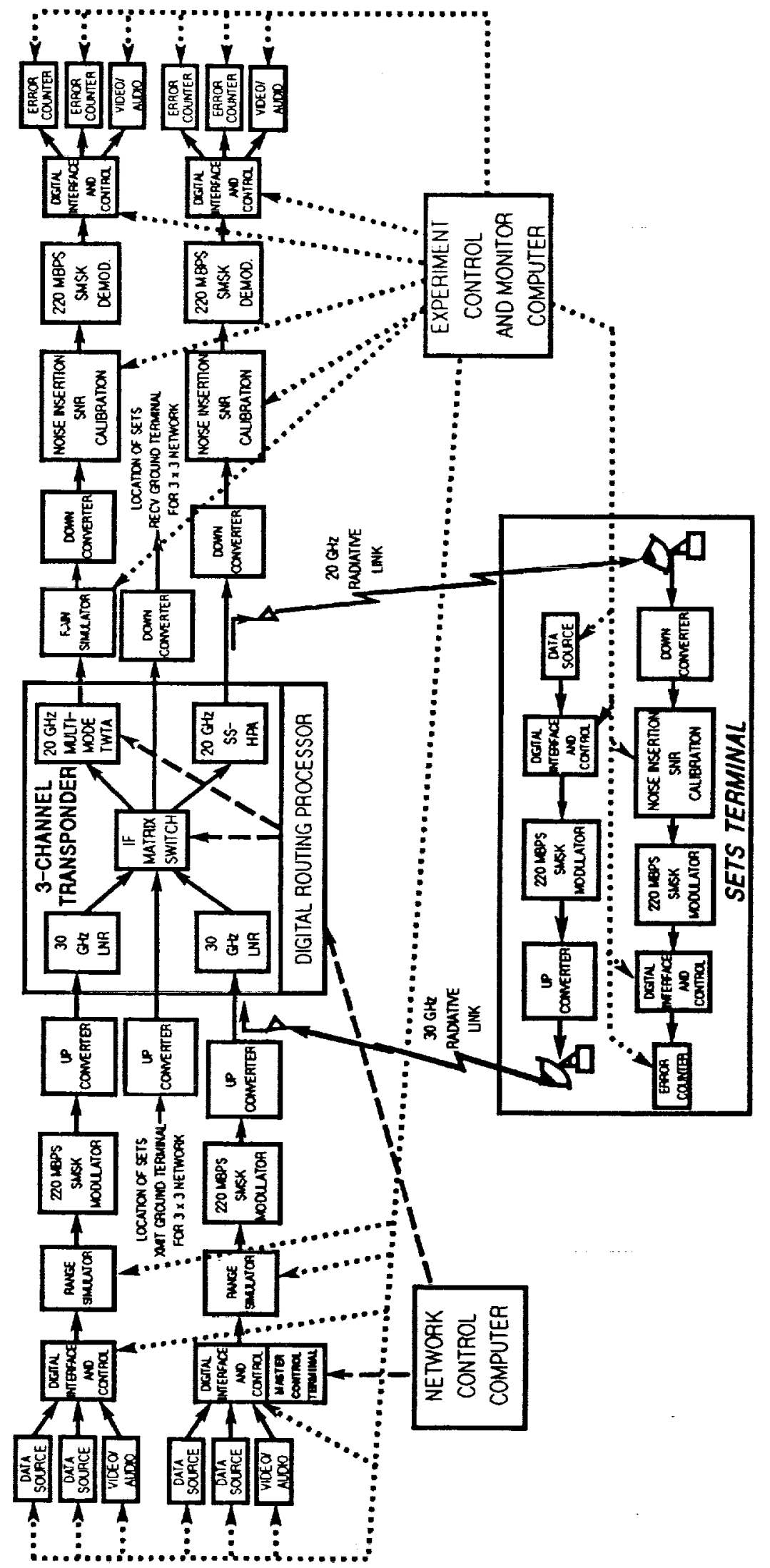

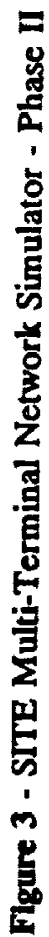


space science experiments, as might occur at the space station or in SEI activities.

The primary project currently underway for this Phase II system is a TDMA network simulation and evaluation ${ }^{18}$. In this program, the performance of a satellite matrix switched TDMA network will be evaluated. The three-channel transponder allows a $3 \mathrm{X}$ 3 network to be evaluated. This is the minimum number of network nodes required for a good simulation. The Master Control Terminal (MCT) must first acquire and track the satellite under varying satellite range, $E_{b} / N_{o}$, fading and interference conditions. The network control must then bring a traffic terminal into the network with complete synchronization under these conditions. Finally, the network must demonstrate the ability to bring in a second traffic terminal and have it communicate with the first traffic terminal, independently of the MCT.

By using the remote terminal, another set of experiments can be performed in which two traffic terminals located within the same spot beam can be brought into the network. The remote terminal can also located in an adjacent frequency channel for interference experiments.

For all aspects of the network simulation, varying combinations of satellite range delay variation, rain attenuation, noise, interference, and signal distortions can be introduced. These experiments, to be carried on for the next two years, are being accomplished concurrently with other component testbed type uses of the facility. Performance measurements for ground terminal hardware, simulation of the ACTS transponder characteristics, and experiments with advanced modems and codecs are planned.

\section{Future Development of the SITE Facility}

The development of a generic test ground terminal will be undertaken within the next year. This ground terminal will allow a variety of modulation formats, coding schemes, and data rates to be interfaced with the SITE facility. Using the same BER measurement and $\mathrm{E}_{\mathrm{b}} / \mathrm{N}_{\mathrm{o}}$ calibration methods as are used currently, several new capabilities will be developed. A comparison of modulation formats and coding schemes as applied to satellite networks as well as the performance of the system at various rates, bandwidths, modulations and codings will be possible. The performance of individual components under these conditions can be evaluated.
Finally, the performance of the modems and codecs themselves, in the presence of noise, interference, and nonlinearity will be measured.

In the course of the SITE facility development, especially in the last few years, the demand for component testing and system-level evaluation and experimentation outside of the planned SITE network simulation has increased. In addition, new uses for the facility and new experiment concepts have been developed by the SITE staff. These demands, and a desire to demonstrate the uses of in-house technology developments, has led to the planning of a second satellite transponder to be used in conjunction with the current hardware. This second transponder will be based on MMIC-based Ka-band hardware developments, and will also be designed to handle higher frequency components and data links in the future. In addition to handling the increasing test and experiment load, the second transponder will have two major uses.

The first use involves the demonstration and evaluation of on-board switching and processing technology currently being developed in-house and under contract at NASA Lewis. This technology development is driven by the need to develop systems with low cost ground terminals for low data rate users. This can be accomplished through narrowband FDMA uplink access architectures which eliminate the need for a high power uplink transmitter. TDMA downlinks can operate in a wideband mode, with the spacecraft transmitter providing adequate power to allow small aperture ground terminals.

In the systems being developed by NASA, the uplink narrowband FDMA traffic, resulting from several spatially isolated uplink beams, will be demodulated by multichannel demultiplexer demodulators (MCDD), one for each uplink beam. The demodulated uplink user data will be switched and routed to proper one of several downlink beam by the information switching processor (ISP). Downlink traffic will be transmitted using high rate modulation in a TDMA format, allowing the satellite high power transmitter to operate at maximum power, thus improving downlink signal-tonoise ratio.

The on-board processing portion such a system is shown in Figure 4. The second transponder will be designed to accommodate the FDMA/TDMA architecture and processing equipment as well as the TDMA/TDMA 


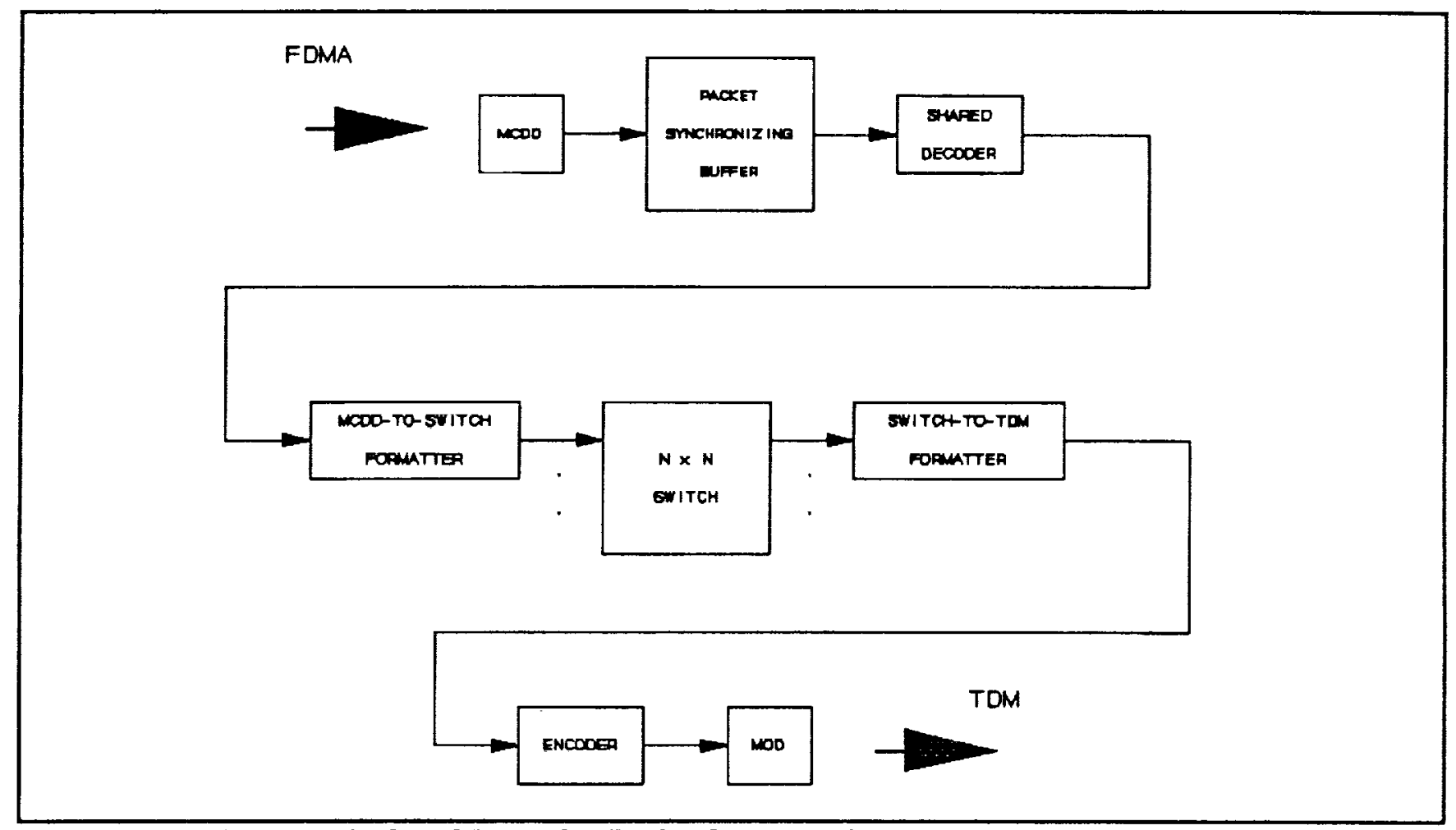

Figure 4 - On-board Processing Portion for FDMA/TDMA Satellite System

architectures. The performance of the system can be assessed in terms of the noise, interference, nonlinearity, rain fade, and satellite range variation parameters, as well as other parameters unique to the FDMA/TDMA architecture, such as intermodulation, adjacent channel interference, and amplitude variation between FDM channels.

The other major use of the second transponder will be as part of an intersatellite link networking simulation. Although still in the conceptual stage, the intersatellite link simulation will allow the investigation of networking concepts for both commercial and space science applications, and the evaluation of components and subsystems designed for such applications. It will allow the current SITE capabilities to remain intact, and will add features unique to intersatellite data relay systems. A conceptual model of the future SITE facility is shown in Figure 5. Given the intended design of the second transponder to allow dual architectures, the networking and interfacing aspects of linking similar as well as dissimilar networks will be examined. The timetable for implementation of these concepts will be driven by the funding availability in the next several years.

\section{Applications to SEI Technologies}

The technology evaluation methods applied in the SITE project to commercial systems are well-suited for application to the long-term development of an SEI communication network. Low noise receivers, TWT and solid state power amplifiers, and microwave switches in the Ka-band region can be evaluated in the current SITE facility. Those which may be developed in other frequency bands can be evaluated with slight modifications to the current facility or in the second transponder. Modems and codecs, data compression hardware, and baseband processing subsystems can also be evaluated in the current version of the facility.

As hardware technology developments become complete, the development method followed for the current SITE simulator can be used to gradually evolve a more and more complex laboratory simulation system designed for the SEI mission. The system would begin with individual link simulators designed to test specific portions of the overall SEI network. Adding other system portions, transponders, intersatellite links and ground stations, a network simulation facility will be developed which will allow complete testing of network 


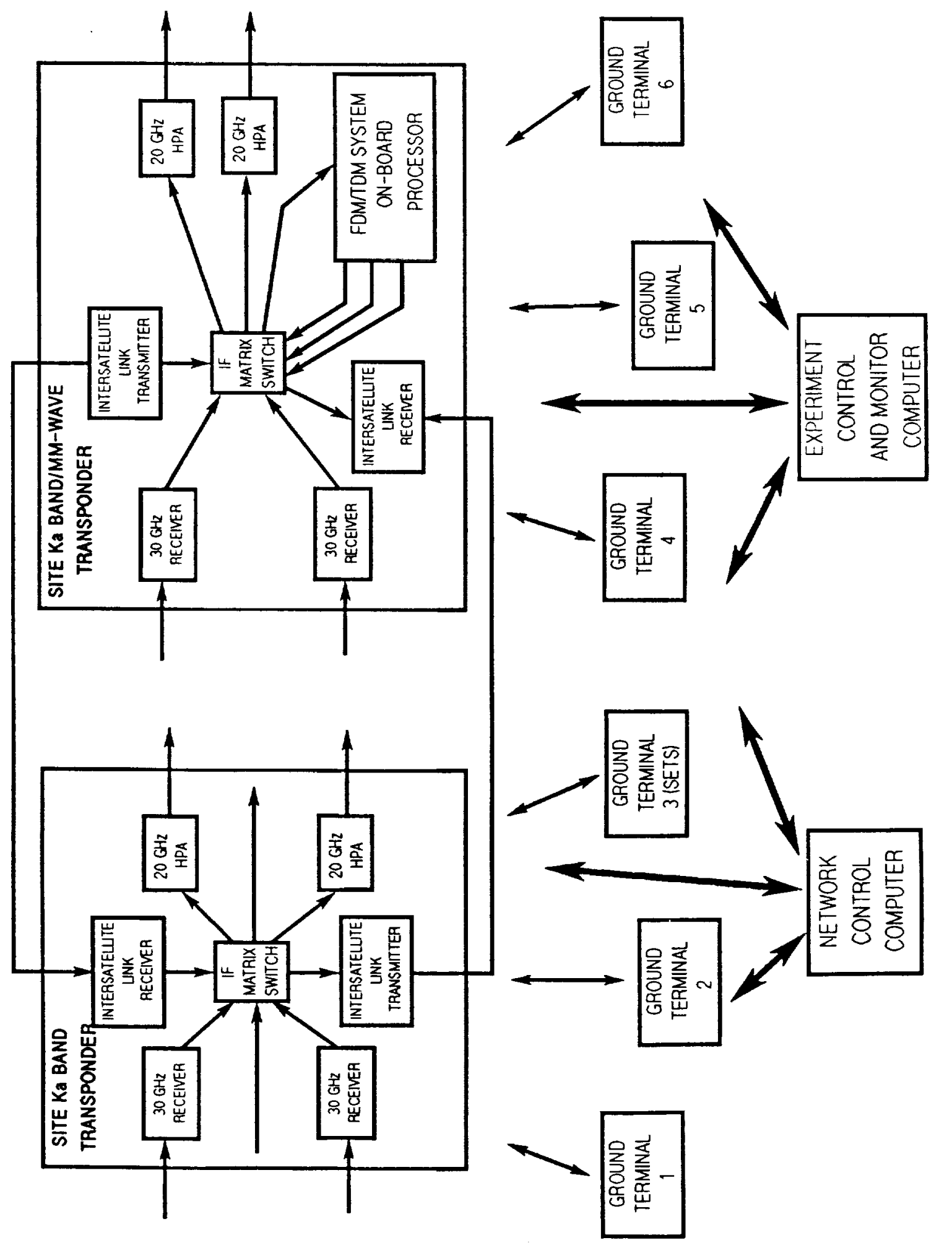

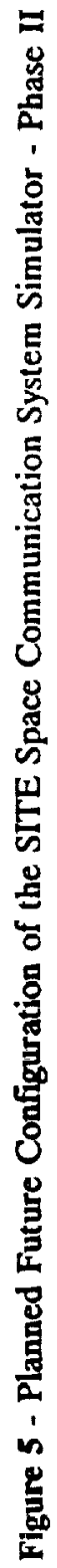


functions, individual systems and subsystems, and components in the presence of complete network control, and system and link impairments. In operating such a network, the delays, delay variations and doppler shifts ultimately become quite large, and system synchronization must be tested with these impairments properly simulated. Finally, the unattended network control architectures, which will be an ongoing parallel development, can be applied to a realistic network for full evaluation and verification.

Ultimately, by combining the hardware and subsystems which will need to be developed individually for the SEI mission, a highly versatile laboratory simulation system, capable of evaluating technologies at the component, subsystem, system, and network level can be developed at a minimum cost. The long-term nature of the SEI mission assures a gradual evolution and expansion of the SEI communication network. Thus, a simulation facility developed in an evolutionary fashion as suggested here will contribute significantly the long-term success of the SEI mission.

\section{Summary}

We have described some of the major development and evaluation requirements for commercial and space science and exploration communications systems of the future. The methods employed for evaluation on the component, subsystem, system, and network level have been described. The SITE Space Communications System Simulator is a test system that has been designed to meet these evaluation requirements, especially for high frequency and high data rate systems. The SITE System has produced significant results in the evaluation and development of components, subsystems, and networks. Constant revision and enhancement of the system is continuing in order to keep pace with expanding technology evaluation requirements. The SEI mission, with its challenging complexity and development requirements, is well-suited for the technology evaluation approach developed in the SITE simulation facility.

\section{REFERENCES}

Ponchak, D. S., Zuzek, J. E., Whyte, W. A. Jr., Spence, R. L., and Sohn, P. Y., "A Technology Assessment of Alternative Communications Systems for the Space Exploration Initiative", 13th AIAA Communication Satellite Systems Conference, March, 1990 (NASA TM 103243).

2 Bagwell, J. W., "A System for the Simulation and Evaluation of Satellite Communication Networks", 10th AIAA Communication Satellite Systems Conference, March, 1984 (NASA TM 83531).

3 Wald, L. W., "Characterization of a $30 \mathrm{GHz}$ IMPATT Solid State Amplifier", NASA Technical Memorandum 100876, July, 1988.

4 Shalkhauser, K. A., and Fujikawa, G., "BitError Rate Testing of High Power $30 \mathrm{GHz}$ Traveling Wave Tube for Ground Terminal Applications", NASA Technical Paper 2365, October, 1986.

5 Kerczewski, R. J., The Bit-Error Rate Performance of a Satellite Microwave Matrix Switch", 12th AIAA Communication Satellite Systems Conference, March, 1988 (NASA TM 100285).

6 Kerczewski, R. J., Ponchak, G. E., and Romanofsky, R. R., "Performance of Five 30 GHz Satellite Receivers", 1989 IEEE MTT International Microwave Symposium", June, 1989 (NASA TM 101960).

7 Kerczewski, R. J., and Fujikawa, G., "Performance Measurements for a LaboratorySimulated $30 / 20 \mathrm{GHz}$ Communication Satellite Transponder", 13th AIAA International Communication Satellite Systems Conference, March, 1990 (NASA TM 102424).

8 Fujikawa, G., and Kerczewski, R. J., "Performance of a Ka-Band Satellite System Under Variable Signal Power Conditions", 1987 IEEE MTT International Microwave Symposium, June, 1987 (NASA TM 88984).

9 Kerczewski, R. J., Fujikawa, G., Svoboda, J. S., and Lizanich, P. J., "Effects of Amplitude Distortion and IF Equalization on Satellite Communication System Bit-Error Rate Performance", 13th AIAA Communication Satellite Systems Conference, March, 1990. (NASA TM 102415). 
10 Kerczewski, R. J., "A Study of the Effect of Group Delay Distortion on an SMSK Satellite Communication Channel", NASA Technical Memorandum 89835, April, 1987.

11 Windmiller, M. J., "Unique Bit-Error Rate Measurement System for Satellite Communications Systems", NASA Technical Paper 2699, March, 1987.

12 Kerczewski, R. J., Daugherty, E. S., and Kramarchuk, I., "Automated Measurement of the Bit-Error Rate as a Function of Signal-toNoise Ratio for Microwave Communication Systems", 29th Automatic RF Techniques Conference, June, 1987 (NASA TM 89898).

13 Shalkhauser, M. J., and Budinger, J. M., "Digitally Modulated Bit-Error Rate Measurement System for Microwave Component Evaluation", NASA Technical Paper 2912, July, 1989.

14 Shalkhauser, M. J., "Satellite Ground Terminal User Simulation", NASA Technical Memorandum 100234, January, 1988.

15 Nagy, L. A., "Satellite Range Delay Simulation for a Matrix Switched Time Division Multiple Access Network Simulation System", 13th AIAA Communication Satellite Systems Conference, March, 1990

16 Shalkhauser, K. A., Nagy, L. A., and Svoboda, J. S. "Rain Fade Simulation and Power Augmentation for Satellite Communication Systems", NASA Technical Memorandum 103134, September, 1990.

17 Fujikawa, G., Conroy, M. J., Saunders, A. L., and Pope, D. E., "Experimental Radio Frequency Link for Ka-Band Communications Applications", NASA Technical Memorandum 100824, June, 1988.

18 Ivancic, W. D., Andro, M., Nagy, L. A., Budinger, J. M., and Shalkhauser, M. J., "Satellite Matrix-Switched Time Division Multiple Access Network Simulation and Evaluation", 13th AIAA Communication Satellite Systems Conference, March, 1990 (NASA TP 2944). 


\section{REPORT DOCUMENTATION PAGE}

Public reporting burden for this collection of information is estimated to average 1 hour per response, Including the time lor reviewing instructions, searching existing data sources, gathering and maintaining the data needed, and completing and revlewing the collection of information. Send comments regarding this burden estimate or any other aspect of this collection of information, including suggestions for reducing this burden, to Washington Headquarters Services, Directorate for information Operations and Reports, 1215 Jefferson Davis Highway, Suite 1204, Arlinglon, VA 22202-4302, and to the Office of Management and Budget, Paperwork Reduction Project (0704-0188), Washington, DC 20503.

1. AGENCY USE ONLY (Leave blanK) 2. REPORT DATE
3. AEPORT TYPE AND DATES COVEAED

Technical Memorandum

\section{TITLE AND SUBTITLE}

Evaluation of Components, Subsystems, and Networks for High Rate, High

Frequency Space Communications

\section{AUTHOR(S)}

Robert J. Kerczewski, William D. Ivancic, and John E. Zuzek

\section{PERFORMING ORGANIZATION NAME(S) AND ADDRESS(ES)}

National Aeronautics and Space Administration

Lewis Research Center

Cleveland, Ohio 44135 - 3191

\section{FUNDING NUMBERS}

WU $-316-30-19$
8. PERFORMING ORGANIZATION REPORT NUMBER

E- 6597

9. SPONSORING/MONITORING AGENCY NAMES(S) AND ADDRESS(ES)

10. SPONSORING/MONITORING AGENCY REPORT NUMBER

National Aeronautics and Space Administration

Washington, D.C. 20546-0001

NASA TM -105274

AIAA - 91 - 3423

\section{SUPPLEMENTARY NOTES}

Prepared for the Conference on Advanced Space Exploration Initiative Technologies cosponsored by AIAA, NASA, and OAI, Cleveland, Ohio, September 4-6, 1991. Responsible person, John E. Zuzek, (216) 433 - 3469.

12. DISTRIBUTION/AVAILABILITY STATEMENT

12b. DISTRIBUTION CODE

Unclassified - Unlimited

Subject Categories 17 and 32

\section{ABSTRACT (Maximum 200 words)}

The development of new space communications technologies by NASA has included both commercial applications and space science requirements. At NASA's Lewis Research Center, methods and facilities have been developed for evaluating these new technologies in the laboratory. NASA's Systems Integration, Test and Evaluation (SITE) Space Communication System Simulator is a hardware-based laboratory simulator for evaluating space communications technologies at the component, subsystem, system, and network level, geared toward high frequency, high data rate systems. The SITE facility is well-suited for evaluation of the new technologies required for the Space Exploration Initiative (SEI) and advanced commercial systems. This paper describes the technology developments and evaluation requirements for current and planned commercial and space science programs. Also examined are the capabilities of SITE, the past, present, and planned future configurations of the SITE facility, and applications of SITE to evaluation of SEI technology.

\section{SUBJECT TERMS}

Space communications; High frequencies; Millimeter waves; Planetary exploration; Testbed; Test facilities; Evaluation

17. SECURITY CLASSIFICATION OF REPORT

Unclassified
18. SECURITY CLASSIFICATION OF THIS PAGE Unclassified
19. SECURTY CLASSIFICATION OF ABSTAACT

Unclassified

\section{NUMBER OF PAGES}

12

16. PRICE CODE

$\mathrm{A} 03$

20. LIMITATION OF ABSTRACT 